\title{
Changes in running speed after incentive shifts
}

\author{
ALLEN H. WOLACH and MARGARET SERES \\ Mlinois Institute of Technology, Chicago, Ill. 60616
}

Rats experienced shifts in reward magnitude after 24,54 , or 108 trials to the first reward magnitude. All Ss were run in a two-way runway situation with six triais every day. As days of preshift training increased, the number of trials before postshift performance shifts occurred also increased. Since Ss with extonded training and only one directional response do not take a long time to shift response rate (Crespi, 1942), the present results were related to the two responses that were required

Crespi (1S.42) and Zeaman (1949) performed studies in which rats experienced shifts in reward magnitude after they approached asymptotic running speeds. They observed that Ss shifted from large to small reward (and from small to large reward) rapidly changed their running speeds to the speeds that were appropriate for the new magnitudes of reward. In addition, the Ss exhibited contrast effects. That is, after two or three postshift trials, Ss shifted from large to small reward ran more slowly than the asymptotic level attained by small-reward Ss before the shift (negative contrast). Similarly, Ss shifted from small to large reward initially ran faster than the preshift rate for large-reward $\mathrm{Ss}$ (positive contrast). Subsequent work has been directed at substantiating the positive and negative contrast effects. The negative contrast effect is well established (e.g., Spence, 1956; DiLollo \& Beez, 1966), while the positive contrast effect is not often obtained (e.g. Spence, 1956; DiLollo $\&$ Beez, 1966). The large number of contrast-effect studies that have been performed tend to obscure the rapid shifts in performance first observed by Crespi (1942). A rapid change in postshift performance is usually taken as an indication that the magnitude of the incentive, rather than a different level of learning, is responsible for the differential running speeds for groups with disparate reward magnitudes in the first phase of the study. If Ss with small reward had learned less in the preshift phase, they would take a relatively long time to change running speeds. Similarly, large-reward Ss would not slow down when shifted to small reward if running speed reflected learning.

Bower (1961) performed a study in which a given $S$ experienced a different magnitude of reward in each of two distinctive runways. These Ss were compared with Ss that experienced only large or small reward in one runway. The $S$ s with differential training ran more slowly in the small-reward runway than did the small-reward Ss (negative contrast). However, the Ss with differential training did not run faster in the large-reward runway than the large-reward Ss; i.e., no evidence for positive contrast was obtained. Beery \& Black (1968) reported a study in which $S$ s received differential training that was similar to the differential training employed by Bower (1961). Half of the Ss in the Beery and Black study experienced a shift in the largeand small-reward runways after 40 trials to each reward magnitude; i.e., the large-reward and the small-reward runways were reversed. The Ss did not produce a rapid change in performance after the shift. The Beery and Black study limits the generality of the observation that $S$ s rapidly attain running speeds that are appropriate to new incentive conditions.

Beery \& Black (1968) suggest that the discrimination that was learned by their Ss was responsible for the slow shift in running speeds. That is, Ss in the Beery and Black study had the opportunity to discriminate the two reward magnitudes in both phases of the study. The possibility for a discrimination is not the only variable that distinguished the simultaneous contrast paradigm (Bower, 1961; Beery \& Black, 1968) from the Crespi (1942) and Zeaman (1949) successive contrast paradigm. The simultaneous contrast approach requires two generic responses, one in each runway. The number of responses, rather than the discrimination, could produce the slow change in postshift performance.

A procedure that is intermediate to the simultaneous and successive contrast paradigms was employed in the present study to clarify differences in rate of response shift in the two paradigms. The Ss experienced only one magnitude of reward in each of the phases (pre- and postshift). However, they had the opportunity to make two responses (running in two directions)

\section{SURJECTS}

Thirty naive 95- to 105-day-old Sprague-Dawley rats served as Ss. The
Ss were housed three to a cage with water available at all times. Two weeks before the initiation of preliminary training, the Ss were placed on a $24 \cdot h$ feeding schedule. During pretraining and the experiment proper, each $S$ was placed in a separate feeding cage immediately after its daily session. Each $S$ was given three $2-\mathrm{cm}$ Wayne Lab Blox pellets and allowed $1 \mathrm{~h}$ to eat before being returned to the home cage.

\section{APPARATUS}

The apparatus was a straight alley runway with a center section $(193.0 \times 12.5 \times 14.5 \mathrm{~cm})$ that was separated from two equal sized end compartments $(27.0 \times 12.4 \times 14.4 \mathrm{~cm}$ ) by guillotine doors. The walls of the runway were made of wood and painted flat black. A steel grid $(1.0 \times 1.0 \times .1 \mathrm{~cm}$ thick) served as the runway floor. The top of the runway was formed by four hinged frames with aluminum screening.

\section{PROCEDURE}

Each $S$ was pretrained on 2 successive days. The $\mathrm{S}$ was placed in the starting end compartment and allowed to explore the runway. Two .045 -g Noyes pellets were placed in the compartment at the opposite end of the runway. When the $S$ entered the compartment that contained the food the guillotine door was closed behind it. The $S$ remained in the compartment until the food was eaten. After $\mathbf{S}$ consumed the food, the door was raised and $S$ was allowed to return to the first end compartment for two additional pellets.

The Ss were assigned randomly to six experimental groups. Half of the Ss (three groups) experienced large reward (five .045-g Noyes pellets) on preshift trials, followed by small reward for the remaining trials (one $.045 \cdot g$ Noyes pellet). Small reward was followed by large reward for the remaining Ss. The three groups that started the study with a given magnitude of reward experienced 4,9 , or 18 days of training before being shifted to the other magnitude of reward. Ten days of postshift training followed the preshift training.

All Ss experienced a two-way running situation with six reinforced trials every day. The Ss started each daily session at the same end of the runway. When a given $S$ reached the opposite end of the runway, the guillotine door was closed behind it for $15 \mathrm{sec}$. After the 15-sec period, the door was raised and the $S$ was required to run to the end compartment that had been the startbox on the first trial. Consequently, the Ss experienced six reinforced trials every day, three in each direction, with a 15-sec postreinforcement delay after every trial. 


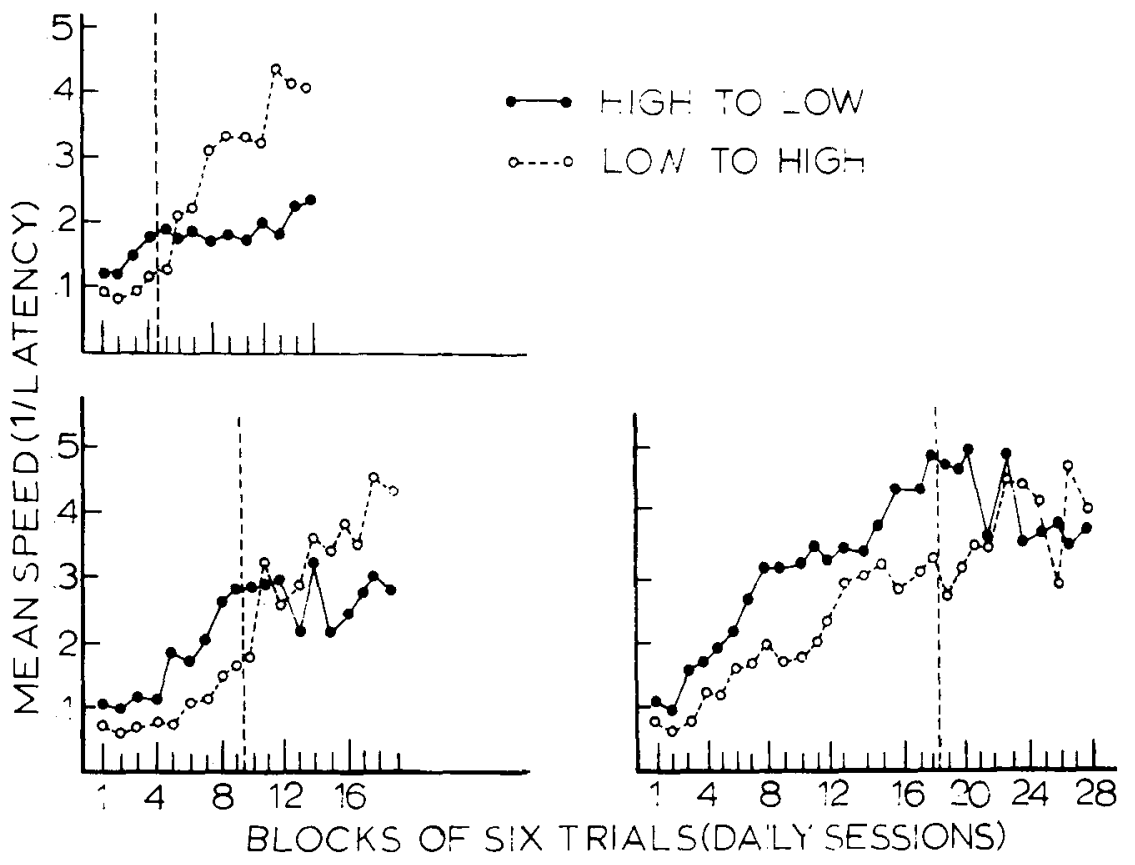

Fig. 1. Pre- and postshift running speeds for Ss with 24, 54, or 108 trials to the first reward magnitude.

Timing of the first trial started when S's feet touched the starting end compartment floor. The trial was terminated when $S$ was $18 \mathrm{~cm}$ into the opposite end compartment and the guillotine door closed behind it, i.e., the time from the placement of the $S$ in the startbox until it reached the opposite end of the runway was recorded. The remaining five trials were initiated by the raising of the guillotine door that confined $S$ in an end compartment and terminated when $S$ was $18 \mathrm{~cm}$ into the opposite end compartment, i.e., the timer registered the time from the opening of a guillotine door until the guillotine door at the opposite end of the runway closed behind the $S$. If an $S$ failed to complete a trial in $90 \mathrm{sec}$, it was gently pushed to the appropriate end of the runway and confined in the end compartment for $15 \mathrm{sec}$ RESULTS

Since the Ss experienced different levels of training in the first phase of the study, the data from groups with different levels of preshift training were analyzed separately. Three analyses of variance (Trial Blocks by Reward Magnitude) were performed on the data from Ss with a given level of preshift training. That is, analyses of variance were performed on the last 4 days (trial blocks) of preshift performance, the first 4 days of postshift performance, and the last 4 days of postshift performance. A Lindquist (1953) Type I analysis of variance was used for each comparison. The data from a $\mathbf{S}$ in a significant Trial Blocks by Reward Magnitude interaction $[F(3,24)=4.12, p<.05]$ for Ss with 4 days of preshift training. The Trial Blocks by Reward Magnitude interaction for the first 4 postshift days was not significant for groups with $9[\mathrm{~F}(3,24)=1.45, \mathrm{p}>.05]$ or 18 $[F(3,24)=1.60, p>.05]$ days of preshift training. Thus, the data reveal that only Ss with the smallest number of preshift trials started to shift running speeds during the 4 days after the switch of reward magnitude.

Postshift running speed (trial blocks) did not increase during the first 4 postshift days for Ss with 4 $[F(3,24)=1.18, \quad p>.05], 9$ $[F(3,24)=1.09, \quad p>.05]$, or 18 $[F(3,24)=.65, p>.05]$ days of preshift training. Similarly, the groups with 4,9 , or 18 days of preshift training did not have significantly different speeds to the two reward magnitudes during the first 4 days of postshift training $[F(1,8)=1.10$, $p>.05 ; \quad F(1,8)=.00, p>.05 ;$ and $\mathrm{F}(1,8)=1.05, \mathrm{p}>.05 ;$ respectively $]$.

The data from the last 4 days of postshift training were analyzed to determine if $S$ acquired running speeds appropriate to the magnitudes of reward they experienced in the second phase of the study. Only the Ss with 4 days of preshift training developed faster running speeds to the large magnitude of reward $[F(1,8)=12.08, \quad p<.05]$. Nine $[F(1,8)=3.20, p>.05]$ or 18 $[F(1,8)=.05, p>.05]$ days of preshift training did not lead to significantly faster running speeds to the large reward. The analyses of variance and the figure indicate that $\mathrm{Ss}$ with extended preshift training did not produce different postshift running speeds by the end of 10 days (60 trials) of postshift training. The running speeds during the last 4 days of postshift training for Ss with 4 days of preshift training were no longer increasing $[F(3,24)=.68, p>.05]$. Running speeds for Ss with 9 days of preshift training $[F(3,24)=3.36$, $\mathrm{p}<.05]$ were still increasing at the end of 10 days of postshift training, while Ss experiencing 18 days of preshift training were no longer increasing in running speed $[F(3,24)=1.36, p>.05]$. The Trial Blocks by Reward Magnitude interaction was not significant for Ss with $4[\mathrm{~F}(3,24)=.91, \mathrm{p}>.05]$ or 9 $[F(3,24)=.88, \quad p>.05]$ days of preshift training. However, Ss experiencing 18 days of preshift training produced a significant Trial Blocks by Reward Magnitude interaction $[\mathrm{F}(3,24)=3.64, \mathrm{p}<.05]$ at the end of postshift training.

\section{DISCUSSION}

The data suggest that Ss with 4 days 
of preshift training started to shift running speeds within 4 days of postshift training, while Ss with 18 days of preshift training were just beginning to shift running speeds at the end of 10 days of postshift training. That is, a small number of preshift trials led to a relatively rapid change in running speed to the two reward magnitudes. More extended training increased the time required for a shift in running speeds. In fact, Ss with 18 days (108 trials) of preshift training and large vs small reward did not have significantly different running rates after 10 days (60 trials) of shifted reward magnitude. It should be noted, however, that even the Ss with 24 preshift trials took considerably longer to shift running speeds than Ss with a comparable number of trials in the Crespi (1942) and Zeaman (1949) studies. Since the Ss did not shift running speeds quickly, it is not surprising that contrast effects were not obtained. The groups with 18 days of preshift training served as controls for the $S s$ that were shifted after fewer days of preshift training. Although no Ss were run with more than 18 days of preshift training, it is obvious that Ss with 18 days of preshift training did not produce a postshift contrast effect. These Ss had not shifted running speeds after 10 days of postshift training. It is unreasonable to expect a successive contrast effect to occur if Ss do not shift running speeds within a few postshift trials. As preshift training trials were increased, more trials were required for Ss to produce postshift changes in performance. This suggests that contrast effects in the present paradigm become less likely as preshift training trials are increased

The present study differed from the paradigm used by Crespi and Zeaman in three major ways. First, the $\mathrm{Ss}$ in four of the groups experienced more trials than the Crespi-Zeaman Ss. Secondly, Ss experienced more daily trials (six as opposed to one) than Crespi and Zeaman employed. Thirdly, two directional responses were required during both phases of the study. Previous studies (Spence, 1956) suggest that number of trials per se is not responsible for the slow change in postshift performance, Spence, using the Crespi-Zeaman paradigm, obtained rapid changes in postshift performance after 48 preshift trials. Zaretski (1966) used the Crespi-Zeaman paradigm with six trials per day. His Ss showed the same rapid shifts in performance that Crespi and Zeaman obtained.

The remaining distinction (number of directional responses in each phase) appears to be responsible for the relatively slow change in postshift performance obtained by Berry \& Black (1968) with a simultaneous contrast design. A given $S$ in the Beery and Black study had the opportunity to respond to two reward magnitudes in two separate runways. Beery and Black posited that the gradual change in postshift performance could be attributed to difficulty in discriminating the shift caused by experiencing two reward magnitudes in the first phase of the study. The present study suggests that the number of responses, rather than an exteroceptive discrimination, is responsible for the slow change in postshift performance.

A response variability explanation (e.g., Schoenfeld, 1968; Ferraro \& Branch, 1968) can account for the present study and the Beery and Black study without making recourse to a discrimination interpretation of the data. One need only assume that each subclass (variant in running topography) of the generic response running in a given direction must be extinguished before postshift response rate can change. The Ss in the Beery and Black study and the present study have the opportunity to acquire more subclass responses than ss experiencing the Crespi-Zeaman paradigm. The greater opportunity for varied subclass responding is generated by the greater number of generic responses (the response in the two running directions or the two runways). Groups with extended training in the first phase of the present study had the opportunity to sample more subclass responses during the first phase of training. The response variability position suggests groups with extended training have more subclass responses to extinguish before shifts in running speed are completed. Therefore, the number of trials required for a shift in running speed should increase with increased training in the first phase of the study. The Crespi-Zeaman one-way runway situation is more likely to produce stereotyped responding than a two-response situation. Stereotyped behavior is not conducive to varied responding. As a consequence, extended training with the Crespi-Zeaman paradigm should be less detrimental to rapid response shifts than extended training with two generic responses.

The present study has several implications for the simultaneous contrast paradigm introduced by Bower (1961). The Ss in Bower's main group experienced two generic responses (one each in the large- and small-reward runway). The two remaining groups (large or small reward) experienced only one runway, and consequently only one generic, response. It is possible that the two single generic response groups are not comparable to the group that made two responses. The $S$ s that experienced only one magnitude of reinforcement should have experienced reinforcements in both runways. This would have contributed to an equal number of generic responses (and similar stimulus conditions) for the three groups. Perhaps the negative contrast effect obtained with Bower's (1961) simultaneous contrast paradigm is an artifact of inappropriate comparison groups.

It has been demonstrated that the two-way runway situation can generate independent response patterns (Wolach, Coutts, \& Aderman, 1970 ) in the two running directions. This makes it possible to perform a successive contrast study in a two-way runway situation with a given direction associated with a given reward magnitude. Control groups would obtain the same reward magnitude in both directions. Such a study would eliminate the possible artifact that Bower had in his design. In addition, it would not be necessary to handle the Ss between daily trials. This would make the design more similar to a free-operant study, and possibly reduce the number of $S s$ needed for stable performance in the three comparison groups.

\section{REFERENCES}

BEERY, R. G.. \& BLACK, R. W. Reversal of magnitude of reward in differential conditioning. Psychological Record, 1968, 18, 179-183.

BOWER, G. H. A contrast effect in differential conditioning. Journal of Experimental Psychology, 1961, 62 . 196-199.

CRESPI, L. P. Quantitative variation in incentive and performance in the white rat. American Journal of Psychology, $1942,55,467-517$.

DiLOLLO, F. D., \& BEEZ, V. Negative contrast effect as a function of magnitude of reward decrement. Psychonomic Science, 1966, 5, 99-100.

FERRARO, D. P., \& BRANCH, K. H. Variability of response location during regular and partial reinforcement. Psychological Reports, 1968, 23, 1023-1031.

LINDQUIST, E. F. Design and analysis of experiments in psychology and education. Boston: Houghton-Mifflin. 1953. Pp. 267-273.

SCHOENFELD, W. N. On the difference in resistance to extinction following regular and periodic reinforcement. Journal of the Experimental Analysis of Behavior, $11,1968,259-261$.

SPENCE, K. W. Behavior theory and conditioning. New Haven: Yale University Press, 1956. Pp. 127-137.

WOLACH, A. H., COUTTS, M., \& ADERMAN, M. Sequenced reinforcement in a two-way runway situation. Psychonomic Science, 1970, 20, 267-269.

ZARETSKY, H. H. Learning and performance in the runway as a function of the shift in drive and incentive. Journal of Comparative \& Physiological Psychology, 1966, 62, 218-221.

ZEAMAN, D. Response latency as a function of the amount of reinforcement. Journal of Experimental Psychology. $1949,39,468-483$ 